Journal of Research in Interprofessional Practice and Education

Vol. 9.1

2019

\title{
Exploring Predictors for Teamwork Performance in an Interprofessional Quality Improvement and Patient Safety Course for Early Learners
}

\author{
Danah M. Alsane a , BsC Pharm, MS, doctoral candidate; Kelly \\ Lockeman $^{\mathrm{a}}$, PhD; Leticia R. Moczygemba ${ }^{\mathrm{b}}$, PharmD, PhD; Colleen

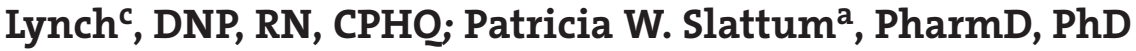

\author{
a. Virginia \\ Commonwealth \\ University \\ b. University of \\ Texas \\ c. HCA Healthcare
}

\section{Journal of Research in Interprofessional Practice and Education (JRIPE) \\ Vol. 9.1 \\ (c) 2019}

doi: 10.22230/jipe.2019 v9n1a283

Corresponding author: Danah M. Alsane. Email: alsanedm@vcu.edu.

\begin{abstract}
Background: This study evaluated predictors of team development and performance on a final project in a large Interprofessional Quality Improvement and Patient Safety course.

Methods and findings: Predictors examined were prior interprofessional teamwork experience and collective orientation preferences for dominance and affiliation. The Team Development Measure assessed perceived level of team development at the end of the course. Structural equation modelling was used to test the relationships, and only dominance was related to team development. Team development was not related to performance on the final project.

Conclusions: This study is the first to simultaneously assess predictors of team development and the relationship between team development and course performance in interprofessional education. Although findings were not conclusive, several avenues for future study are highlighted.
\end{abstract}

Keywords Interprofessional education; Collaboration; Teamwork; Team development

\section{Introduction}

Teams and teamwork is an important core competency for interprofessional education (IPE) and collaborative practice [1]. Interprofessional education is an approach that allows students to work with learners in other health disciplines during their training in order to better prepare them for collaborative practice. In designing an IPE activity, health professions educators should focus on incorporating and evaluating teamwork. Teamwork assessment allows for the evaluation of how individuals' contributions influence team function and helps to identify areas where there is potential for improvement [2,3].

Existing IPE literature has used a variety of approaches to evaluate teamwork performance, using both faculty observation and student self-reporting. The use of dif- 
2

Teamwork Performance Predictors in IPE for Early Learners

Alsane, Lockeman, Moczygemba, Lynch, Slattum
Journal of Research in Interprofessional Practice and Education

Vol. 9.1

2019 ferent instruments allows for the examination of various dimensions and aspects of teamwork. Several common instruments for the evaluation of teamwork have been applied in an IPE context, particularly in experiential IPE settings, including TeamSTEPPS and the McMaster-TOSCE scale $[4,5,6,7,8]$. The use of tools that involve direct observation requires training and is resource intensive. These tools also fail to examine the factors that may be affecting team performance, particularly those that relate to the individual members who comprise the team. This is problematic since teamwork literature suggests that collective orientation can predict teamwork performance in tasks that require decision-making, negotiating, and executing skills [9]. Studies in healthcare settings have also found a correlation between team development and team performance $[10,11,12]$.

The main objectives of this study were to explore how individual characteristics affect team development and to assess the relationship between team development and team performance in an IPE course. This contributes to the literature in IPE by extending the study of teamwork from the experiential setting to the didactic environment. In addition, this study uses data collected at the individual level to predict group-level outcomes, a novel analytical approach that has not been examined in prior IPE literature. This represents an important area of study because the ability to predict group outcomes from individual characteristics may offer a less resourceintensive way to study team development and its influence on team performance.

\section{Methods}

This study used a cross-sectional design. Measures collected from medical, pharmacy, and nursing students during educational activities associated with a large introductory IPE course comprised several measures. Additional data were collected using an electronic questionnaire embedded in the course evaluation survey that was administered at the end of the semester.

\section{Course description}

Interprofessional Quality Improvement and Patient Safety (IPQIPS) is a required one-credit, pass/fail course that was offered for the first time in spring 2016. This course replaced the program-specific introductory patient safety curricula for prelicensure students in medicine, nursing, and pharmacy and introduced students to quality improvement from an interprofessional perspective. Throughout the course, health professions students collaborated within interprofessional teams to apply the course content to structured learning activities. Class met weekly and included activities such as lectures, talks by guest speakers, practical application exercises, and case-based learning. Activities were designed to enable students to examine the complexity of the healthcare system, learn commonly used safety design standards, evaluate hazards and common causes of healthcare errors, design interventions to improve the quality of healthcare, and examine the approaches for designing and sustaining a culture of safety.

Enrolled students $(n=498)$ were divided into 88 interprofessional teams; each team was composed of five to six students. Class met once a week for 75 minutes. 
Teamwork Performance Predictors in IPE for Early Learners

Alsane, Lockeman, Moczygemba, Lynch, Slattum
Journal of Research in Interprofessional Practice and Education

\section{Journal of Research in Interprofessional Practice and Education}

Teams were distributed throughout four learning studios (large classrooms designed for team-based learning), with 22 teams in each room, and instruction occurred simultaneously. In each learning studio, a team of three faculty members delivered instruction and facilitated learning activities. The faculty teams included health professions educators and practicing clinicians with expertise in quality improvement and patient safety science; each profession (medicine, nursing, and pharmacy) was represented in each learning studio. To ensure consistency in instruction across different learning studios, common instructional materials were used, and faculty held a formal meeting before each weekly class session to discuss the session content. Learning assessment methods included a quiz, a midterm exam, and a final project.

\section{Conceptual framework}

This study is framed around an input-process-outcome model for predicting team effectiveness [13]. This framework suggests that the individual characteristics of team members have an effect on group processes, which in turn influence the effectiveness of the team. The hypothesis was that teams comprised of members with a higher propensity for working in a collective manner [9] and more prior experience working on interprofessional teams (the inputs) would demonstrate greater team development (the process), and the interaction between team development and content knowledge measured by quiz scores (a mediator) would predict better performance on team-based course assignments (the output). Figure 1 presents a theoretical model representing this hypothesis.

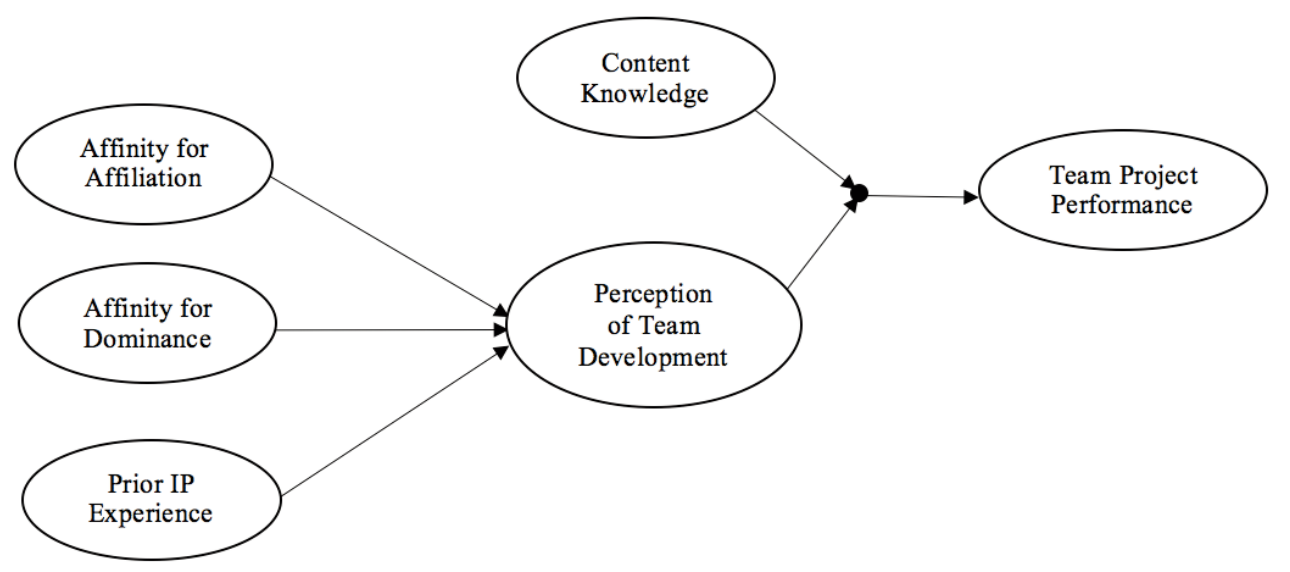

Figure 1: Theoretical Model

\section{Measures and data collection}

Multiple measures were used to examine the study hypotheses. Two of the research measures (an individual knowledge quiz and a team-based final project) were required assessments for students and teams participating in the course. At the end of the semester, a course evaluation survey was distributed electronically using survey software (Qualtrics, Provo, UT) [14] to all enrolled students, and additional research measures were included in the questionnaire. The Virginia Commonwealth University (VCU) review board approved this study as exempt, and students were 
Teamwork Performance Predictors in IPE for Early Learners

Alsane, Lockeman, Moczygemba, Lynchy, Slattum

Journal of Research in Interprofessional Practice and Education

\section{Journal of Research in Interprofessional Practice and Education}

informed that their participation in the survey was voluntary and would not affect their course grade. During the final class session, course instructors encouraged students to participate in the survey, which was available for ten days. During this period, students who had not yet completed the survey were sent reminder emails on up to three occasions.

\section{Content knowledge}

A knowledge quiz was administered to individual students during the ninth class session. It was scored on a percent-correct basis. Higher scores indicated a greater understanding of the course material required for the effective completion of the final project. The quiz was created by the course director, refined using feedback from course faculty, and approved by all instructors. Content validity based on expert opinion was the primary criteria for the use of the quiz as a measure in this study, and the measure was considered a mediating variable because the collective knowledge of team members may have had an interaction effect on the process of team development.

\section{Team performance}

The final project had to be completed by each team by the eleventh class session and required students to apply their individual knowledge and teamwork skills toward the successful completion of an assignment as a team. Students were asked to apply what they had learned to define, measure, analyze, improve, and control interventions to improve acute stroke care. The project consisted of two components: an Ishikawa diagram worth 15 points and a written report worth 25 points. Similar to the quiz, the instructional team created the content and requirements for the assignment. Each project was graded by one of four instructors using a standard rubric. To maximize consistency, the four instructors met to identify and discuss grading outliers before finalizing project scores. Scores for each component were then summed and converted to a percent scale.

\section{Team development}

To collect data about each team's perceived level of development, the Team Development Measure (TDM) [15] was included in the course evaluation survey. The TDM is a 31-item measure grounded in teamwork literature and extensively tested in clinical workplace settings. While it has not been formally validated in classroom-based teams, it is the closest tool available for measuring the process that occurs when collaborative student teams work together to complete complex casebased assignments related to interprofessional care. The TDM has a total possible score ranging from 0-100 and was administered at an individual level. A higher score represents individual experiences consistent with involvement in a more highly developed team [15].

\section{Affiliation and dominance}

The 15-item Collective Orientation Scale [9], also included with the course evalua- 
5

Teamwork

Performance

Predictors in IPE for

Early Learners

Alsane, Lockeman, Moczygemba, Lynch, Slattum
Journal of Research in Interprofessional Practice and Education tion survey, was used to assess the affinity for affiliation and dominance of individuals within teams. This measure was designed specifically to gauge individual differences that have been linked to team task performance, and validity evidence has been reported based on content, scale structure, and relationship with other scales. Each item is measured on a five-point Likert scale. The affiliation subscale includes 10 items that measure an individual's preference for working with others versus working alone; the dominance subscale, which measures self-interest, dominance, and control versus interest in others and cooperation, includes the remaining five items. Higher scores indicate a higher affinity for each measure [9].

\section{Prior interprofessional experience}

Prior interprofessional teamwork experience was measured using a single question with four response choices: "How many times in the past have you worked on an interprofessional team that included students or clinicians from other health professions? (1) Never, (2) Once or twice, (3) Several times, (4) Many times." Demographic data were linked to the survey data from student records maintained by the university. These variables included the student's academic major, gender, age, and race/ethnicity.

\section{Statistical analysis}

Descriptive statistics and preliminary analyses were carried out using SAS software, version 9.4 (SAS Institute, Cary NC) [16]. These included characteristics of the sample and comparisons between students by program and team performance by instructor using one-way analysis of variance (ANOVA) and Chi-square analysis. The main analysis was conducted using Mplus software, version 7 [17]. Structural equation modelling was used to test the study hypotheses and control for clustering by grading instructors.

Because all study measures except the final project score (representing team performance) were collected at the level of the individual student, there were two options for analysis: a) aggregate the individual-level measures for each team, or b) take a latent variable approach and use the individual-level measures to model the team score for each measure. Aggregating the individual-level measures has been shown to result in biased estimates [18], so the latent variable model approach was selected for model testing. Analysis also required a multilevel approach to account for clustering within instructor. Because there were missing data at the individual level, each latent variable was examined independently to ensure that it performed appropriately before the final structural equation model was fit to test the hypothesis.

\section{Results}

Descriptive statistics

\section{Response rate}

The sample consisted of 498 students who completed the IPQIPS course. Overall, 299 students (60\%) completed the TDM measure, 315 (63\%) completed the affiliation subscale of the collective orientation measure, 309 (62\%) completed the domi- 
JRIPE

6

Teamwork Performance Predictors in IPE for Early Learners

Alsane, Lockeman, Moczygemba, Lynch, Slattum

nance subscale, and 311 (62\%) responded to questions regarding interprofessional teamwork experience. Response rates varied by discipline, with completion rates for medical and nursing students ranging from 65-70 percent depending on these measures, while response rates for pharmacy students were lower at 44-45 percent. The knowledge quiz was mandatory, so data on this measure were available for all 498 students. Similarly, all 88 teams completed the mandatory final project measuring team performance. The mean age of participating students was $25(S D=3.7)$, and the median response to the question about prior experience working on an interprofessional team was 3 ("Several times") with an interquartile range of 2-3.

\section{Demographic characteristics}

Of the 498 students enrolled in the course, 216 (43\%) were medical students in an MD program, 149 (30\%) were pursuing a baccalaureate degree in nursing, and 133 (27\%) were pharmacy students in a PharmD program. The majority of students (59\%) were female, and 54 percent were white.

\section{Preliminary findings}

\section{Individual measures}

The analysis of variance revealed significant differences in individual student responses by program on affinity for affiliation, $F(2,312)=3.21, p=0.041$, and perceptions of team development, $F(2,296)=5.57, p=0.004$. Responses about prior interprofessional teamwork experience also showed variation between students of different programs, $\chi^{2}(6, N=311)=48.8, p<0.001$. There were no differences by program in individual responses on the measure for dominance or on content knowledge. Descriptive statistics for each measure are displayed in Table 1.

Table 1: Descriptive statistics for individual measures

Journal of Research in Interprofessional Practice and Education

Vol. 9.1

2019

\section{Table 1: Descriptive statistics for individual measures}

\begin{tabular}{|l|c|c|c|c|}
\hline & Medicine & Nursing & Pharmacy & Overall \\
\hline & Mean (SD) & Mean (SD) & Mean (SD) & Mean (SD) \\
\hline Affinity for affiliation* & $3.11(0.55)$ & $3.00(0.53)$ & $3.21(0.52)$ & $3.09(0.54)$ \\
\hline Affinity for dominance & $2.6(0.62)$ & $2.76(0.66)$ & $2.62(0.81)$ & $2.66(0.68)$ \\
\hline Perception of team development* & $61(10.1)$ & $56(9.5)$ & $58(13.3)$ & $59(10.8)$ \\
\hline Content knowledge & $85(15.0)$ & $83(16.6)$ & $83(16.6)$ & $84(15.9)$ \\
\hline & Medicine & Nursing & Pharmacy & 0verall \\
\hline & $n(\%)$ & $n(\%)$ & $n(\%)$ & $n(\%)$ \\
\hline Prior experience on an IP Team* & & & & \\
\hline Never & $53(35 \%)$ & $4(4 \%)$ & $4(7 \%)$ & $61(20 \%)$ \\
\hline Once or twice & $23(16 \%)$ & $25(24 \%)$ & $15(25 \%)$ & $63(20 \%)$ \\
\hline Several times & $41(28 \%)$ & $44(43 \%)$ & $28(47 \%)$ & $113(36 \%)$ \\
\hline Many times & $31(21 \%)$ & $30(29 \%)$ & $13(22 \%)$ & $74(24 \%)$ \\
\hline
\end{tabular}

*Variance between programs for these measures was significant at $p<0.05$. 
Teamwork Performance Predictors in IPE for Early Learners

Alsane, Lockeman, Moczygemba, Lynch, Slattum
Journal of Research in Interprofessional Practice and Education

\section{Team performance}

Scores on the final project were negatively skewed and ranged from 28.5 to 40 $(M=37.1, S D=2.19)$. Analysis of variance revealed a significant effect on final project scores due to different instructors grading the final projects, $F(3,84)=12.5$, $p<0.001$. Based on this variance, model testing for the main analysis controlled for clustering by instructor.

\section{Model testing}

Software limitations in Mplus precluded the display of indicators for overall model fit and significance, while simultaneously controlling for clustering by instructor. Since variance by instructor had already been identified as an issue with the team performance measure, it was deemed more important to control for the variance than to test the overall fit for the hypothesized model. Instead, the individual regression results were reviewed for each path within the model. Thus, Hypothesis 1 is that the collective affiliation, dominance, and prior interprofessional teamwork experience among team members predict their collective perceptions of team development. Hypothesis 2 is that the interaction between a team's collective perception of team development and its collective content knowledge predicts team performance on the final project. For each path, the parameter estimates for each predictor were examined using an alpha of $p<0.05$ as an indication of significance. Results are presented in Table 2. For Hypothesis 1, when collective affiliation, dominance, and interprofessional teamwork experience were examined together, only dominance was found to have a significant relationship with team development. For Hypothesis 2, the interaction between team development and collective knowledge measured by quiz scores did not have a significant relationship with team performance, despite controlling for the instructor effect.

Table 2: Parameter estimates for each predictor in the theoretical model

\begin{tabular}{|l|c|c|}
\hline & Estimate (SE) & $p$ \\
\hline Hypothesis 1 & & \\
\hline Affiliation & $-7.09(6.07)$ & 0.243 \\
\hline Dominance & $13.94(5.15)$ & 0.007 \\
\hline Prior interprofessional teamwork experience & $2.49(5.12)$ & 0.626 \\
\hline Hypothesis 2 & & \\
\hline Interaction of team development x knowledge & $0.02(0.01)$ & 0.113 \\
\hline
\end{tabular}

\section{Discussion}

This cross-sectional study examined the effect of team development on level of performance in completing a team-based project. Using the same data, it also evaluated the predictors associated with variance in team development. The simultaneous evaluation of both aspects is a strength of this study, as previous studies have tended to examine only one of the questions in isolation [4]. 
8

Teamwork Performance Predictors in IPE for Early Learners

Alsane, Lockeman, Moczygemba, Lynch, Slattum

Journal of Research in Interprofessional Practice and Education

Vol. 9.1

2019
Two hypotheses were tested in the current study. The first hypothesis was that student teams with more favourable collective scores on measures of affiliation (higher), dominance (lower), and interprofessional teamwork experience (higher) would exhibit more advanced levels of team development. Although the results were not significant for affiliation or interprofessional teamwork experience, higher levels of dominance were significantly associated with better teamwork development. The significant positive relationship between the higher level of dominance and TDM scores is inconsistent with the study hypothesis, but it may have a logical interpretation in a leadership context. Leadership may be associated with dominance. As a result, teams that show a higher affinity for dominance might include more individual team members who possess strong leadership skills. Strong leaders may contribute to effective team development by motivating team members to function effectively within the team to accomplish team goals, improve communication and trust among team members, and help team members to understand their roles and responsibilities [19,20,21].

The second hypothesis was that scores on the final team-based final project would be predicted by the interaction between team development and the collective knowledge that the team members demonstrated related to the project topic. The results did not show a statistically significant relationship; however, other studies have found evidence supporting such a relationship, both in an IPE context [4] and in more general studies of team performance in healthcare settings [22]. This study's non-significant finding could have several explanations.

First, the TDM was designed and tested on clinical teams in healthcare settings, and it was used here as a measure to evaluate team development in a classroombased team experience. There has been no validity evidence for its use in a didactic setting. Second, the current study assessed the relationship between team development and performance, with the score on a final course project as the measure for team performance. Course instructors designed the assignment and grading rubric based on the content presented in the course, but the measure has not been examined for evidence of construct validity. Third, the TDM examines four elements of team development: cohesion, communication, clarity of team roles, and clarity of team goals [15]. Here, the total scale was used as a measure of overall team development in the sample without examining these subscales, and its relationship was evaluated with subscales of the Collective Orientation Scale. The findings suggest that while there is some association, particularly on the dominance subscale, the Collective Orientation Scale does not fully account for the team development results. It is possible that the context of the didactic course was not suitable for team development on all of those dimensions. Furthermore, since the team performance measure (final project score) has not been tested for validity or examined in depth, it is not certain that this task resembled the team-based tasks previously used in studies related to team development. Additionally, design and statistical issues discussed in the limitations section could have influenced the study.

\section{Study limitations}

One major challenge of this study was the potential for differential patterns in miss- 
9

Teamwork Performance Predictors in IPE for Early Learners

Alsane, Lockeman, Moczygemba, Lynch, Slattum
Journal of Research in Interprofessional Practice and Education

Vol. 9.1

2019 ing data. Although some measures were mandatory and thus had a 100 percent response rate, all other measures were optional and had lower response rates. Response rates also varied by student program, which may contribute to error. Another major challenge of this study was the potential for confounding by factors external to the hypothesis. Student scores on the measures for affiliation, interprofessional teamwork experience, and team development were found to differ by student program. There were also differences in student knowledge and clinical experience by program, as medical students were in the foundation stage of their program, while nursing and pharmacy students were in more advanced stages. This introduces the potential for student program to act as a confounder, since it was not accounted for in the model. Finally, scores on the final team projects were homogenous and highly skewed. The lack of overall variation in the outcome measure may have limited the ability to effectively evaluate the hypothesis. Despite the use of a rubric and grading discussions to minimize scoring inconsistency, there was significant variation in final project grading between instructors, but only within the small overall range of scores. An attempt was made to account for this variation by controlling for instructor in the analyses, but these differences may also have posed limitations to model testing.

\section{Future studies}

Further studies of team dynamics and development in an IPE context are recommended, particularly as related to team performance outcomes. The current study focuses on evaluating the role of individual characteristics on team development. Additional studies are needed to evaluate the effect of group and institutional characteristics on team development. Results of such studies can help educators determine which factors are important for facilitating the development of effective teams in a didactic setting. In addition, more studies that measure predictors of teamwork and evaluate team development in IPE settings are needed. The current study used the Collective Orientation Scale to evaluate individual characteristics that relate to team performance, and it used the TDM to assess team development. Other studies may attempt to replicate the use of these two measures in a setting that avoids some of the limitations of the current study, making it possible to further evaluate the use of such measures in didactic IPE settings. It could also be valuable to evaluate the use of other measures, both for the predictors of performance and for the measurement of team development. Results of such studies will help health professions educators focus on such factors and emphasize the role of teamwork in designing IPE activities. Such studies could also help to develop and validate methods for the assessment of these constructs in a didactic IPE setting.

\section{Conclusion}

This study adds to the IPE literature examining the role of collective orientation in predicting team development. In addition, it examined team development as a predictor of team performance in an IPE setting. This is the first study to assess both relationships simultaneously. Although the limitations of this study prevent draw- 
10

Teamwork Performance Predictors in IPE for Early Learners

Alsane, Lockeman, Moczygemba, Lynch, Slattum
Journal of Research in Interprofessional Practice and Education

Vol. 9.1

2019 ing definitive conclusions, the finding that a collective affinity for dominance among team members was positively associated with team development is interesting and worthy of replication. Future studies that address the current limitations are needed to improve an understanding about the role of group orientation in team development and the role of team development in improving task outcomes in IPE settings.

\section{Acknowledgements}

The authors would like to thank Dr. Robert Perera in the department of biostatistics at VCU for his assistance in the data analyses and interpretation.

\section{References}

1. Interprofessional Educational Collaborative. (2016). Core competencies for interprofessional collaborative practice: 2016 update. Washington, DC: Interprofessional Education Collaborative, 1-10.

2. Buring, S.M, Bhushan, A., Brazeau, G., Conway, S., Hansen, L., \& Westberg S. (2009). Keys to successful implementation of interprofessional education: Learning location, faculty development, and curricular themes. American Journal of Pharmaceutical Education, 73(4), 1-11. doi:10.5688 /aj730460

3. Thistlethwaite, J. (2012). Interprofessional education: A review of context, learning and the research agenda. Medical Education, 46(1), 58-70. URL: http://www.ncbi.nlm.nih.gov/pubmed /22150197. doi:10.1111/j.1365-2923.2011.04143.x [February 9, 2015].

4. Blue, A., Kern, D., Shrader, S., \& Zoller, J. (2013). Interprofessional teamwork skills and attitudes as predictors of clinical outcomes in a simulated learning setting. Journal of Interprofessional Care, 27, 161.

5. Lie, D., May, W., Richter-Lagha, R., Forest, C., Banzali, Y., \& Lohenry, K. (2015). Adapting the McMaster-Ottawa scale and developing behavioral anchors for assessing performance in an interprofessional Team Observed Structured Clinical Encounter. Medical Education Online, 20, 26691. doi:10.3402/meo.v20.26691

6. Ekmekci, O., Plack, M., Lelacheur, S., Lewis, K., \& Schlumpf, K. (2015). Assessing performance and learning in interprofessional health care teams. Journal of Allied Health, 44(4), 236-243.

7. Emmert, M.C., \& Cai, L. (2015). A pilot study to test the effectiveness of an innovative interprofessional education assessment strategy. Journal of Interprofessional Care, 29(5), 451-456. doi: $10.3109 / 13561820.2015 .1025373$

8. Dobson, R.T., Stevenson, K., Busch, A., Scott, D.J., Henry, C., \& Wall, P.A. (2009). A quality improvement activity to promote interprofessional collaboration among health professions students. American Journal of Pharmaceutical Education, 73(4). doi:10.5688/aj730464

9. Driskell, J.E., Salas, E., \& Hughes, S. (2010). Collective orientation and team performance: Development of an Individual Differences Measure. Human Factors: The Journal of the Human Factors and Ergonomics Society, 52(2), 316-328. doi:10.1177/0018720809359522

10. Valentine, M.A., Nembhard, I.M., \& Edmondson, A.C. (2012). Measuring teamwork in health care settings: A review of survey instruments. Medical Care. 53(4), 16-30. doi:10.1097/MLR $.0 \mathrm{~b} 013 \mathrm{e} 31827$ feef6

11. Clements, D., Dault, M., \& Priest, A. (2007). Effective teamwork in healthcare: Research and reality. Healthcare Papers, 7, 26-34. doi:10.12927/hcpap.2013.18669

12. Manser, T. (2009). Teamwork and patient safety in dynamic domains of healthcare: A review of the literature. Acta Anaesthesiologica Scandinavica, 53(2), 143-151. doi:10.1111/j.1399-6576.2008 .01717.x

13. Guzzo, R.A., \& Dickson, M.W. (1996). Teams in organizations: Recent research on performance and effectiveness. Annual review of psychology, 47(1), 307-338.

14. Qualtrics [Computer software]. (2016). URL: https://www.qualtrics.com Provo, UT: Qualtrics.

15. Stock, R., Mahoney, E., \& Carney, P.A. (2013). Measuring team development in clinical care settings. Family Medicine, 45(10), 691-700.

16. SAS (Version 9.4) [Computer software]. (2014). URL: https://www.sas.com . Cary, NC: The SAS Institute.

17. Muthén, L. K., \& Muthén, B. O. (2017). Mplus user's guide: Eighth edition. Los Angeles, CA: Muthén \& Muthén. 


\section{JRIPE \\ Journal of Research in Interprofessional Practice and Education}

11

Teamwork

Performance

Predictors in IPE for

Early Learners

Alsane, Lockeman, Moczygemba,

Lynch, Slattum
18. Croon, M., \& van Veldhoven, M.J.P.M. (2007). Predicting group-level outcome variables from variables measured at the individual level: A latent variable multilevel model. Psychological Methods, 12(1), 45-57. doi:10.1037/1082-989X.12.1.45

19. Elkins, T., \& Keller, R.T. (2003). Leadership in research and development organizations: A literature review and conceptual framework. Leadership Quarterly, 14(4-5), 587-606. doi:10.1016 /S1048-9843(03)00053-5

20. NHS Leadership Academy. (2009). Leadership Framework Organisational Toolkit: The LF and Coaching 1, 1-2.

21. Stewart, G.L. (2006). A meta-analytic review of relationships between team design features and team performance. Journal of Management, 32(1), 29-55. doi:10.1177/0149206305277792

22. Wheelan, S.A., Burchill, C.N., \& Tilin, F. (2003, November 1). The link between teamwork and patients' outcomes in intensive care units. American Journal of Critical Care, 12(6), 527-534. 